\title{
Enhancement of invadopodia activity in glioma cells by sublethal doses of irradiation and temozolomide
}

\author{
Leon Mao, BSc, ${ }^{1}$ Clarissa A. Whitehead, BSc, ${ }^{1}$ Lucia Paradiso, ${ }^{1}$ Andrew H. Kaye, MD, ${ }^{1,2}$ \\ Andrew P. Morokoff, PhD, ${ }^{1,2}$ Rodney B. Luwor, PhD, ${ }^{1}$ and Stanley S. Stylli, PhD ${ }^{1,2}$ \\ 1Department of Surgery, The University of Melbourne, The Royal Melbourne Hospital; and 2Department of Neurosurgery, The \\ Royal Melbourne Hospital, Parkville, Victoria, Australia
}

\begin{abstract}
OBJECTIVE Glioblastoma is the most common primary central nervous system tumor in adults. These tumors are highly invasive and infiltrative and result in tumor recurrence as well as an extremely poor patient prognosis. The current standard of care involves surgery, radiotherapy, and chemotherapy. However, previous studies have suggested that glioblastoma cells that survive treatment are potentially more invasive. The goal of this study was to investigate whether this increased phenotype in surviving cells is facilitated by actin-rich, membrane-based structures known as invadopodia.
\end{abstract}

METHODS A number of commercially available cell lines and glioblastoma cell lines obtained from patients were initially screened for the protein expression levels of invadopodia regulators. Gelatin-based zymography was also used to establish their secretory protease profile. The effects of radiation and temozolomide treatment on the glioblastoma cells were then investigated with cell viability, Western blotting, gelatin-based zymography, and invadopodia matrix degradation assays.

RESULTS The authors' results show that the glioma cells used in this study express a number of invadopodia regulators, secrete MMP-2, and form functional matrix-degrading invadopodia. Cells that were treated with radiotherapy and temozolomide were observed to show an increase primarily in the activation of MMP-2. Importantly, this also resulted in a significant enhancement in the invadopodia-facilitated matrix-degrading ability of the cells, along with an increase in the percentage of cells with invadopodia after radiation and temozolomide treatment.

CONCLUSIONS The data from this study suggest that the increased invasive phenotype that has been previously observed in glioma cells posttreatment is mediated by invadopodia. The authors propose that if the formation or activity of these structures can be disrupted, they could potentially serve as a viable target for developing novel adjuvant therapeutic strategies that can be used in conjunction with the current treatment protocols in combatting the invasive phenotype of this deadly disease.

https://thejns.org/doi/abs/10.3171/2017.5.JNS17845

KEY WORDS glioma; invadopodia; invasion; radiotherapy; temozolomide; oncology

$\mathrm{M}$ ALIGNANT gliomas account for approximately $2.3 \%$ of all cancer-related deaths in the US, with more than 22,000 new patients expected annually. ${ }^{17}$ Glioblastoma (GBM, WHO Grade IV) is recognized as the most prevalent form of glioma, ${ }^{28}$ with a median survival of about 15 months, despite standard treatment with surgery, fractionated radiotherapy, and the DNA-alkylating agent temozolomide (TMZ). ${ }^{34}$ Despite this multidisciplinary approach resulting in a modest increase in survival, only $26.5 \%$ of individuals remain alive at 2 years. ${ }^{35} \mathrm{~A}$ critical characteristic of all gliomas is their ability to migrate away from the primary point of tumor origin and extensively infiltrate the surrounding normal brain parenchyma, which hinders the surgical aspect of treatment when removing or ablating all malignant cells is attempted. ${ }^{14,35}$ In addition, resistance to radiotherapy and

ABBREVIATIONS ECM = extracellular matrix; EGFR = epidermal growth factor receptor; FITC = fluorescein isothiocyanate; GBM = glioblastoma; MMP = matrix metalloproteinase PBS = phosphate-buffered saline; TCGA = The Cancer Genome Atlas; TMZ = temozolomide .

SUBMITTED April 4, 2017. ACCEPTED May 23, 2017.

INCLUDE WHEN CITING Published online November 17, 2017; DOI: 10.3171/2017.5.JNS17845. 
TMZ has been well documented, contributing to the poor survival outcome. ${ }^{18}$

It is known that a property shared by many types of tumor cells that possess high invasive or metastatic potential is the ability to form membrane-located structures known as invadopodia. ${ }^{7,26,36,37}$ These are dynamic actin-dependent protrusions that can adhere to and subsequently proteolytically degrade extracellular matrix (ECM) substrates via the action of various transmembrane and secreted extracellular proteases. $7,26,37$ There is a potential role for invadopodia-mediated invasion in glioma, as these structures have been detected in both glioma cell lines and primary tumor cells isolated from ex vivo-cultured GBM specimens. ${ }^{10,37}$ It has also been reported that matrix metalloproteinases (MMPs) that facilitate invadopodia-mediated tumor cell invasion are also upregulated in cancers, including GBM, compared with their normal cell counterparts. ${ }^{42,44,48}$

As radiotherapy has been the main form of treatment prior to TMZ, studies have revealed that this treatment can influence tumor invasion through the induced secretion of a number of factors, including MMPs and vascular endothelial growth factor. ${ }^{21,49}$ Importantly, there is evidence that sublethal doses of radiation can promote glioma invasion, which is associated with enhanced MMP-2 levels in the treated areas ${ }^{30,46}$ and has similarly been observed in a number of different cancers. ${ }^{9,15}$ This is a critical observation, as the majority of GBMs inevitably recur close to either the resection cavity or the target volume of radiotherapy. ${ }^{19}$ In addition, an increase in expression of various proteases, including MMPs, has been shown after TMZ treatment. ${ }^{12}$ As MMPs facilitate the ECM degradation ability of invadopodia, it is conceivable that the increased MMP expression and invasiveness of glioma cells observed after radiotherapy, TMZ treatment, or a combination of both might be mediated by invadopodia. Here, we examine the effects of treatment on invadopodia activity in glioma cells after treatment, with the hope that new therapeutic strategies can be developed in the future by targeting invadopodia-mediated glioma cell invasion.

\section{Methods}

\section{Oncomine Data Mining}

Oncomine (version 4.5, www.oncomine.org; Compendia Bioscience, part of Life Technologies) was used to perform differential analyses on the mRNA expression levels of invadopodia-related regulators in glioma tissue. Oncomine is an online cancer and normal tissue microarray database containing 729 data sets (91,866 samples). These data sets are compiled from publically available cancer microarray data, which are then processed using the same criteria before being made available. ${ }^{32}$ Glioma data sets that were deposited into the Oncomine database were used to examine the mRNA expression levels of various invadopodia regulators in GBM compared with normal brain tissue.

\section{Cell Lines and Cell Culture}

U118, U138, U87MG, LN18, and LN229 human GBM cell lines were purchased from ATCC (American Type Culture Collection). Cell lines \#4, \#15, \#28, \#35, \#39, and \#41 were generated from biopsy samples obtained in GBM patients at the time of surgery at The Royal Melbourne Hospital. The Melbourne Health Research Ethics Committee provided approval for generating the in-house GBM cell lines. The cells were cultured in DMEM (Life Technologies) supplemented with $10 \%$ (vol/vol) heat-inactivated fetal bovine serum (HyClone), penicillin (100 U/ $\mathrm{ml})$, and streptomycin $(10 \mu \mathrm{g} / \mathrm{ml})$. They were maintained in a humidified atmosphere of $10 \% \mathrm{CO}_{2}$ at $37^{\circ} \mathrm{C}$ and used within the first 20 cell passages.

\section{Cell Viability}

Cells were plated in 96-well plates $\left(5 \times 10^{3} / 100 \mu \mathrm{l}\right)$ and allowed to adhere overnight. Triplicate plates were treated with different doses of radiotherapy $(0,2.5$, or $5 \mathrm{~Gy})$ or treated with $\operatorname{TMZ}(0,100$, and $1000 \mu \mathrm{M})$ in triplicate. The combinatorial approach involved treatment first with the relevant dose of radiotherapy followed by a 6-hour incubation of the cells at $37^{\circ} \mathrm{C} / 10 \% \mathrm{CO}_{2}$ in a humidified environment prior to the subsequent addition of TMZ. The cells were incubated for an additional 72 hours at $37^{\circ} \mathrm{C} / 10 \%$ $\mathrm{CO}_{2}$ in a humidified environment and then lysed, and the cell viability relative to a vehicle control was determined on a bioluminometer using a commercially available Cell Titer-Glo kit (Promega) following the manufacturer's instructions.

\section{Invadopodia Matrix Degradation and Colocalization Assays}

Coverslips coated with fluorescein isothiocyanate (FITC)-conjugated gelatin were prepared as described previously. ${ }^{39}$ For the invadopodia profile screen, $5 \times 10^{4}$ cells were seeded per coverslip and incubated at $37^{\circ} \mathrm{C} / 10 \%$ $\mathrm{CO}_{2}$ in a humidified environment for 24 hours. To detect the actin puncta of the invadopodium core, TRITC-phalloidin (dilution 1:75, Sigma-Aldrich) was used, and the total number of cells per image was determined via DAPI nuclear staining (5 $\mu \mathrm{g} / \mathrm{ml}$, Sigma-Aldrich). Confocal microscopy images were acquired using a Nikon A1+ confocal microscope system equipped with a Plan Apo VC 60x DIC N2 oil immersion objective. Degraded FITC-labeled gelatin was defined as a black area depleted of fluorescent gelatin within each acquired image. A total of 10-15 random image fields were captured for every cell line/experimental condition. Images were saved in both the Nikonnative format and as an uncompressed TIFF file and analyzed using ImageJ (version 1.51f; NIH). Threshold and region tools were used to define total regions of matrix degradation in a given image field, and the area of degradation was computed using the particle counter function and normalized with respect to the number of cells (DAPIpositive nuclei). Examination of the effect of radiotherapy and TMZ on the invadopodia activity of GBM cells was investigated as follows: subconfluent cells were treated with a single radiotherapy dose of $5 \mathrm{~Gy}$ and then incubated for 8 hours at $37^{\circ} \mathrm{C} / 10 \% \mathrm{CO}_{2}$ in a humidified environment, and TMZ was added at a final concentration of $1000 \mu \mathrm{M}$. The cells were then incubated for an additional 24 hours at $37^{\circ} \mathrm{C} / 10 \% \mathrm{CO}_{2}$ in a humidified environment, at which point the cells were washed with phosphate-buffered sa- 
line (PBS), trypsinized, and then seeded $5 \times 10^{4}$ cells per well on FITC-conjugated gelatin-coated coverslips and incubated at $37^{\circ} \mathrm{C} / 10 \% \mathrm{CO}_{2}$ in a humidified environment for 24 hours.

\section{Gelatinase Zymography}

For gelatinase zymographic analyses, cells were allowed to adhere overnight and were then washed twice with sterile PBS (pH 7.4) and subsequently incubated in serum-free OptiMEM (ThermoFisher Scientific) for 24 hours for the various experimental conditions as outlined in the relevant figures. A 100- $\mu$ l aliquot was isolated and centrifuged for 10 minutes at $1000 \mathrm{~g}$, and the supernatants were stored at $-20^{\circ} \mathrm{C}$ for subsequent analyses. Gelatinase zymography assaying was performed using Novex $10 \%$ Zymogram (gelatin) protein gels (Life Technologies) to determine the MMP proteolytic activity of the GBM cell lines. Conditioned serum-free OptiMEM media (100 $\mu \mathrm{l} /$ sample) was isolated from each treatment and prepared for electrophoresis under nonreducing conditions. Sample loading was normalized against the concentration of the corresponding cell protein lysates, which were determined by the BCA protein assay (Pierce, ThermoFisher Scientific). The gels were separated by electrophoresis at $125 \mathrm{~V}$ for 1.5 hours in $1 \times$ Novex tris-glycine sodium dodecyl sulfate running buffer and were subsequently incubated in a $1 \times$ Novex zymogram renaturing buffer for 30 minutes (all ThermoFisher Scientific). This was followed by a 30-minute incubation in a $1 \times$ Novex zymogram developing buffer that was replaced with new developing buffer and incubated overnight at $37^{\circ} \mathrm{C}$. The gels were then washed in distilled water before being stained for 1 hour in SimplyBlue Stain (Life Technologies) before further washing in distilled water until clear gelatinolytic bands were visible and their molecular weight was identified against the loaded Precision Blue (BioRad) protein markers. The gels were then scanned on a flatbed scanner for densitometric analysis using ImageJ. The effect of a single treatment of radiotherapy $(0,2.5$, and $5 \mathrm{~Gy})$ or $\operatorname{TMZ}(0,200$, and $2000 \mu \mathrm{M})$ on the MMP profile of the GBM cell lines was carried out 48 hours posttreatment (at 24 hours posttreatment, serum containing medium was removed and cells were washed with PBS, which was replaced with serumfree OptiMEM). The combined treatment of radiotherapy $(0,2.5$, and $5 \mathrm{~Gy})$ and TMZ $(1000 \mu \mathrm{M})$ was carried out as follows: cells were treated with radiotherapy and then incubated at $37^{\circ} \mathrm{C} / 10 \% \mathrm{CO}_{2}$ in a humidified environment for 8 hours, and then $1000 \mu \mathrm{M}$ TMZ was added to the serum-free OptiMEM for an additional 24 hours, at which time an aliquot of conditioned medium was taken.

\section{Western Blot}

Protein lysates $(15 \mu \mathrm{g})$ were subjected to electrophoresis on NuPage $4 \%-12 \%$ bis-tris precast gels (Invitrogen) and blotted on nitrocellulose membrane (GE Healthcare). The normalized protein amounts were analyzed by immunoblotting with the following antibodies: cortactin (diluted 1:1000, Santa Cruz Biotechnology), phospho-cortactin (diluted 1:1000, Cell Signaling Technologies), GAPDH (diluted 1:1000, Cell Signaling Technologies), Nck1 (diluted 1:1000, Cell Signaling Technologies), N-WASP (diluted 1:1000, Santa Cruz Biotechnology), MMP-2 (diluted 1:1000, Santa Cruz Biotechnology), MMP-9 (diluted 1:1000, Santa Cruz Biotechnology), and Tks5 (diluted 1:1000, Santa Cruz Biotechnology). Prior to overnight incubation with primary antibodies, the membranes were blocked with $3 \%$ bovine serum albumin in $1 \%$ TBST for 1 hour at room temperature. Immunoblots were subsequently probed with the appropriate secondary horseradish peroxidase-conjugated antibody (BioRad) and developed with the use of the enhanced chemiluminescence reagent (GE Healthcare) and Super RX x-ray film (Fujifilm).

\section{Results}

\section{Invadopodia Regulator mRNA Expression Is Elevated in GBM Relative to Normal Brain Tissue}

We systematically examined the mRNA gene expression levels of a number of invadopodia-related regulators in GBM tissue and compared the expression levels with those of normal brain tissue using brain tumor data sets; both data sets were obtained from the Oncomine database (Table 1). The $\mathrm{p}$ values were determined using the Student t-test; $\mathrm{p}<0.05$ was considered to indicate a statistically significant result. We observed that various invadopodia regulators were overexpressed in GBM tissue compared with normal brain. Of the 9 regulators listed in Table 1, the overexpression of multiple regulators within 1 data set were observed in a number of the 9 studies (Sun et al., ${ }^{40}$ $\mathrm{n}=5$ regulators; Bredel et al., ${ }^{6} \mathrm{n}=4$; Murat et al., ${ }^{25} \mathrm{n}=4$; Liang et al., ${ }^{22} \mathrm{n}=3$; The Cancer Genome Atlas [TCGA; https://cancergenome.nih.gov, $n=3$; and Shai et al. ${ }^{33} \mathrm{n}=$ 2). Furthermore, examination of the overexpression prevalence of the regulators showed that Nck1 and MMP-2 were overexpressed in 7 and 5 studies, respectively, followed by Tks4 (4 studies) and Tks5, Grb2, and Src (2 studies each). The increased mRNA expression of invadopodia regulators in GBM tissue relative to normal brain in these data sets supports our previous evidence associating invadopodia presence with glioma cells. ${ }^{36,37}$

\section{GBM Cell Lines Express Invadopodia Regulators, Secrete MMP-2, and Form Functional FITC-Labeled Gelatin-Degrading Invadopodia}

In an analogy to our previous study, ${ }^{36}$ in which we had demonstrated that an invadopodia regulator (Tks5) had shown prognostic potential in GBM patients, data mining of the Oncomine glioma data sets in the current study showed increased mRNA expression of a number of regulators in GBM tissue (Table 1). We also examined the protein expression levels of a number of these invadopodia regulators in our GBM cell lines. As outlined in Fig. 1, a number of key proteins were selected for screening by Western blot analyses, including Tks5, cortactin, phospho-cortactin, Nck, N-WASP, and MMP-2. These proteins have been shown to play a role in the initiation, formation, and activity of invadopodia.,26,37 The protein expression levels were varied across the different cell lines. Total cortactin was present at high levels across the majority of the cell lines, as were Nck, N-WASP, and MMP-2.

Tumor cell invasion mediated by invadopodia activity encompasses several processes, including the recruitment 
TABLE 1. Invadopodia regulator mRNA is overexpressed in GBM relative to normal brain tissue

\begin{tabular}{|c|c|c|c|c|c|c|c|c|c|c|}
\hline $\begin{array}{l}\text { Invadopodia } \\
\text { Marker }\end{array}$ & $\begin{array}{l}\text { Cancer } \\
\text { Tissue } \\
\text { Sample }\end{array}$ & $\begin{array}{c}\text { No. of } \\
\text { Samples }\end{array}$ & $\begin{array}{l}\text { Corresponding } \\
\text { Tissue Sample }\end{array}$ & $\begin{array}{c}\text { No. of } \\
\text { Samples }\end{array}$ & $\begin{array}{c}\text { Total } \\
\text { Measured } \\
\text { Genes }\end{array}$ & $\begin{array}{c}\text { Mean Fold } \\
\text { Change } \\
\left(\log _{2}\right)\end{array}$ & $p$ Value & $\begin{array}{l}\text { Sample } \\
\text { Type }\end{array}$ & Platform & Study \\
\hline Cortactin & GBM & 542 & Normal brain & 10 & 12,624 & 1.353 & 0.003 & mRNA & $\begin{array}{c}\text { Human Genome } \\
\text { Y133A Array }\end{array}$ & TCGA \\
\hline Grb2 & GBM & 22 & Normal brain & 3 & 19,574 & 1.348 & $4.20 e-02$ & mRNA & $\begin{array}{l}\text { Human Genome U133 } \\
\text { Plus 2.0 Array }\end{array}$ & Lee et al., 2006 \\
\hline Grb2 & GBM & 27 & Normal brain & 7 & 8,603 & 1.701 & $2.75 e-04$ & mRNA & $\begin{array}{l}\text { Human Genome } \\
\text { U95A-Av2 Array }\end{array}$ & Shai et al., 2003 \\
\hline MMP-2 & GBM & 27 & Normal brain & 4 & 14,836 & 6.426 & $5.41 e-04$ & mRNA & ND & Bredel et al., 2005 \\
\hline MMP-2 & GBM & 30 & Normal brain & 3 & 9,957 & 4.537 & $3.00 e-03$ & mRNA & ND & Liang et al., 2005 \\
\hline MMP-2 & GBM & 80 & Normal brain & 4 & 19,574 & 2.92 & $2.98 \mathrm{e}-04$ & mRNA & $\begin{array}{l}\text { Human Genome U133 } \\
\text { Plus 2.0 Array }\end{array}$ & Murat et al., 2008 \\
\hline MMP-2 & GBM & 81 & Normal brain & 23 & 19,574 & 3.548 & $7.99 e-16$ & mRNA & $\begin{array}{l}\text { Human Genome U133 } \\
\text { Plus 2.0 Array }\end{array}$ & Sun et al., 2006 \\
\hline MMP-2 & GBM & 542 & Normal brain & 10 & 12,624 & 4.818 & $4.06 e-10$ & mRNA & $\begin{array}{c}\text { Human Genome } \\
\text { U133A Array }\end{array}$ & TCGA \\
\hline Nck1 & GBM & 30 & Normal brain & 2 & 9,957 & 1.626 & $1.90 e-02$ & mRNA & ND & $\begin{array}{l}\text { Beroukhim et al., } \\
2007\end{array}$ \\
\hline Nck1 & GBM & 27 & Normal brain & 4 & 14,836 & 1.717 & $1.00 e-02$ & mRNA & ND & Bredel et al., 2005 \\
\hline Nck1 & GBM & 30 & Normal brain & 3 & 9,957 & 1.626 & $1.90 e-02$ & mRNA & ND & Liang et al., 2005 \\
\hline Nck1 & GBM & 80 & Normal brain & 4 & 19,574 & 1.885 & $5.00 e-03$ & mRNA & $\begin{array}{l}\text { Human Genome U133 } \\
\text { Plus 2.0 Array }\end{array}$ & Murat et al., 2008 \\
\hline Nck1 & GBM & 27 & Normal brain & 7 & 8,603 & 1.377 & $3.40 e-07$ & mRNA & $\begin{array}{l}\text { Human Genome } \\
\text { U95A-Av2 Array }\end{array}$ & Shai et al., 2003 \\
\hline Nck1 & GBM & 81 & Normal brain & 23 & 19,574 & 1.305 & $5.41 e-07$ & mRNA & $\begin{array}{l}\text { Human Genome U133 } \\
\text { Plus 2.0 Array }\end{array}$ & Sun et al., 2006 \\
\hline Nck1 & GBM & 542 & Normal brain & 10 & 12,624 & 2.056 & $4.06 \mathrm{e}-09$ & mRNA & $\begin{array}{c}\text { Human Genome } \\
\text { U133A Array }\end{array}$ & TCGA \\
\hline Nck2 & GBM & 80 & Normal brain & 4 & 19,574 & 1.135 & $2.00 e-03$ & mRNA & $\begin{array}{l}\text { Human Genome U133 } \\
\text { Plus 2.0 Array }\end{array}$ & Murat et al., 2008 \\
\hline N-WASP & GBM & 81 & Normal brain & 23 & 19,574 & 1.338 & $1.10 e-02$ & mRNA & $\begin{array}{l}\text { Human Genome U133 } \\
\text { Plus 2.0 Array }\end{array}$ & Sun et al., 2006 \\
\hline Src & GBM & 80 & Normal brain & 4 & 19,574 & 1.035 & $4.50 e-02$ & mRNA & $\begin{array}{l}\text { Human Genome U133 } \\
\text { Plus 2.0 Array }\end{array}$ & Murat et al., 2008 \\
\hline Src & GBM & 81 & Normal brain & 23 & 19,574 & 1.601 & $2.00 e-03$ & mRNA & $\begin{array}{l}\text { Human Genome U133 } \\
\text { Plus 2.0 Array }\end{array}$ & Sun et al., 2006 \\
\hline Tks4 & GBM & 27 & Normal brain & 4 & 14,836 & 3.257 & $1.12 e-04$ & mRNA & ND & Bredel et al., 2005 \\
\hline Tks4 & GBM & 30 & Normal brain & 3 & 9,957 & 1.492 & $1.40 e-02$ & mRNA & ND & Liang et al., 2005 \\
\hline Tks4 & GBM & 80 & Normal brain & 4 & 19,574 & 2.241 & $1.32 \mathrm{e}-06$ & mRNA & $\begin{array}{l}\text { Human Genome U133 } \\
\text { Plus 2.0 Array }\end{array}$ & Murat et al., 2008 \\
\hline Tks4 & GBM & 81 & Normal brain & 23 & 19,574 & 2.194 & $2.50 \mathrm{e}-04$ & mRNA & $\begin{array}{l}\text { Human Genome U133 } \\
\text { Plus 2.0 Array }\end{array}$ & Sun et al., 2006 \\
\hline Tks5 & GBM & 27 & Normal brain & 4 & 14,836 & 1.399 & 0.048 & mRNA & ND & Bredel et al., 2005 \\
\hline Tks5 & GBM & 22 & Normal brain & 7 & 7,689 & 1.263 & 0.005 & mRNA & ND & $\begin{array}{l}\text { Yamanaka et al., } \\
2006\end{array}$ \\
\hline
\end{tabular}

ND = not defined.

mRNA expression levels of invadopodia regulators were examined in GBM and normal brain tissue within the Oncomine database. Displayed in this table are the mean fold changes versus normal brain in each study and overall p-value in that data set. Gene expression data are log transformed and normalized as previously described. ${ }^{39}$

of inactive proenzymes (pro-MMPs) to the invadopodial tip followed by the subsequent secretion and activation (active MMPs), leading to MMP-mediated degradation of the surrounding ECM. This enzymatic activity can be ana- lyzed by gelatin-based zymography, as can be seen in Fig. 2 . Our zymographic analyses of conditioned serum-free medium from the glioma cells at 24 hours revealed that the prominent MMP forms identified were pro-MMP-2 


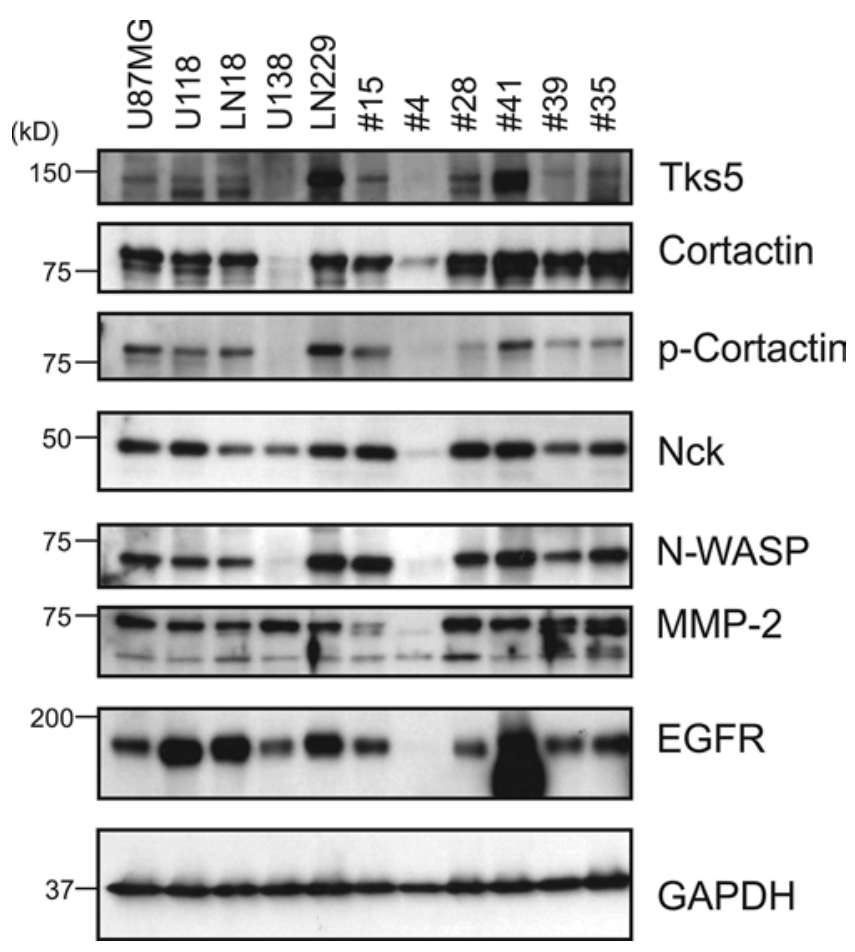

FIG. 1. GBM cells express invadopodia regulator proteins. Immunoblot analysis of various regulator proteins of invadopodia formation or activity (Tks5, cortactin, p-cortactin, Nck, N-WASP, MMP-2, EGFR, and GAP$\mathrm{DH})$ in our screen of $11 \mathrm{GBM}$ cell lines. The experiment was repeated twice, and representative images are shown.

(72 kD) and active MMP-2 (65 kD). Also, as observed on Western blotting (Fig. 1) with MMP-specific antibodies, total MMP-2 protein was detectable in the GBM cell protein lysates, while MMP-9 expression was extremely low or absent. The presence of invadopodia regulator proteins in the Western blot analysis coupled with the detection of secreted MMP-2 activity (Fig. 2) in the culture medium indicates the potential for invadopodia matrix-degrading potential in these glioma cells.

For the remainder of the study, we focused on 3 of the commercially available GBM cell lines (U118, U87MG, and LN229). While these cell lines possess similar intracellular MMP-2 protein levels, as determined by Western blotting, they were observed to express different levels of invadopodia-related regulators, such as Tks5, cortactin, phospho-cortactin, and N-WASP (Fig. 1). In addition, the most frequent genetic alteration associated with GBM is amplification of the EGFR gene, which results in overexpression of epidermal growth factor receptor (EGFR) ${ }^{23}$ and has also been observed to facilitate invadopodia activity through the downstream phosphorylation of invadopodia regulators. ${ }^{16}$ EGFR expression levels of the different GBM cell lines are also indicated in Fig. 1. Importantly, the presence of a number of the invadopodia regulator proteins coupled with MMP-2 zymographic activity can further be associated with the ability of these GBM cell lines to form functional matrix-degrading invadopodia (Fig. 3). These cells exhibit differing degrees of FITC-labeled gelatindegrading invadopodia as depicted by the presence of actin puncta labeled with rhodamine phalloidin that colocalize within areas of the degraded gelatin. The presence of cells with actin puncta and no corresponding area of gelatin degradation potentially signify the early stages of actin recruitment for invadopodia initiation and formation (Stages I and II) prior to the recruitment of MMPs during the mature and late invadopodia stages (Stages III and IV) that result in matrix degradation at the invadopodial tip. ${ }^{1}$ Furthermore, we were also able to demonstrate colocalization of the invadopodia regulators cortactin and Tks5 with actin puncta, confirming the presence of functional gelatin-degrading invadopodia as seen in the representative images of the LN229 cells (Fig. 4).

\section{GBM Cell Lines Exhibit Varying Cell Viability in Response to Radiotherapy or TMZ Treatment}

Prior to examining the effect of radiotherapy or TMZ on invadopodia, we used the 3 GBM cell lines (U118, U87MG, and LN229), to establish a cell viability profile in response to treatment with radiotherapy or TMZ. To examine their sensitivity to radiotherapy, we irradiated the cells at different doses $(0,2.5$, and 5 Gy). As can be seen in Fig. 5A, a differential response is evident, with the U118 and LN229 cells showing increased sensitivity in a dose-dependent manner compared with the U87MG GBM cells. The sensitivity of the 3 glioma cell lines to TMZ was next examined at 3 concentrations: 0,100 , and $1000 \mu \mathrm{M}$ (Fig. 5B). The U118 and U87MG glioma cell lines displayed a similar response to the increasing TMZ concentrations, whereas the LN229 cells exhibited an increased level of resistance. Collectively, these results demonstrate that the TMZ and radiotherapy doses used in this study are sublethal doses and do not result in complete loss of viable cells.

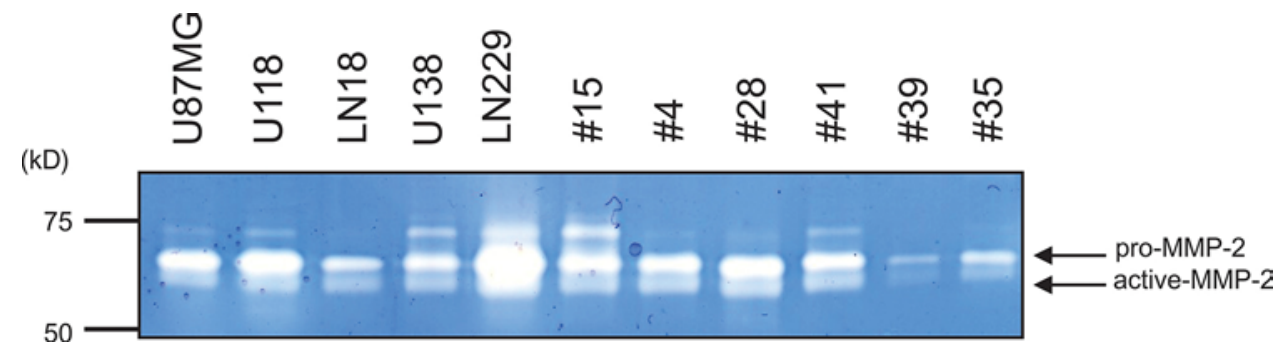

FIG. 2. GBM cells secrete MMP-2. Gelatin zymogram analysis showing MMP-2 activity at 24 hours after incubation of GBM cells in serum-free OptiMEM medium. The experiment was repeated twice, and a representative image is shown. Figure is available in color online only. 


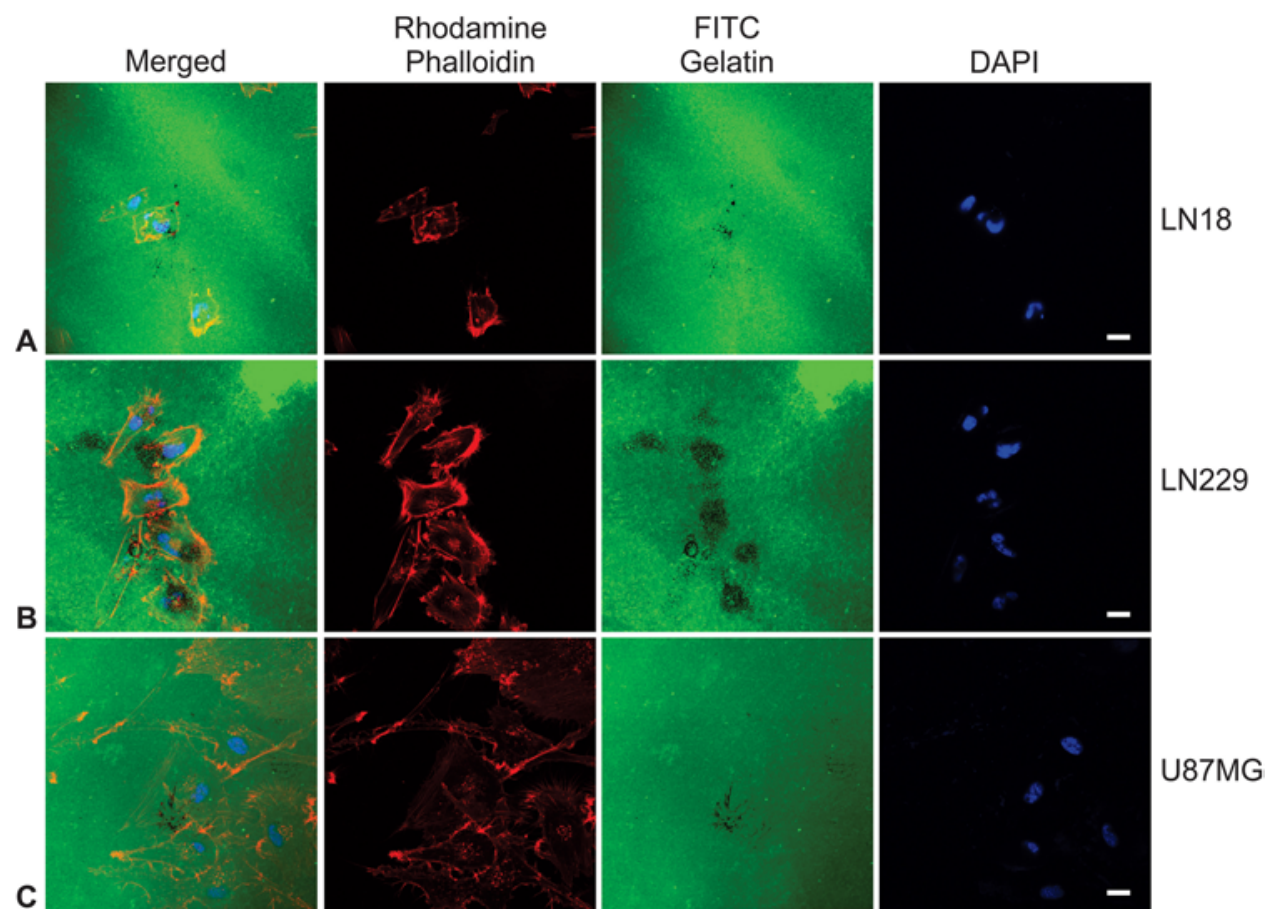

FIG. 3. GBM cells form functional matrix-degrading invadopodia. LN18, U87MG, and LN229 GBM cells were plated on coverslips coated with a thin film of cross-linked FITC-labeled gelatin. After 24 hours, the cells were fixed and stained for actin filaments with rhodamine-phalloidin (red) and DAPI as a nuclear stain. Degraded areas of FITC-labeled gelatin are evident as black areas devoid of FITC-labeled gelatin (green). DAPI staining of the nucleus is shown in blue. The experiment was repeated twice and representative images are shown. Scale bar $=20 \mu \mathrm{m}$.

\section{Radiation and TMZ Treatment Can Enhance MMP-2 Secretion and Activation}

Models of invadopodia formation and activity have been proposed by a number of groups ${ }^{1,26}$ and involve a number of stages (Stage I, early precursor or initiation stage; Stage II, late precursor or pre-invadopodia; Stage III, early mature invadopodia; and Stage IV, late mature invadopodia), which encompass the invadopodia core structure formation, maintenance of actin polymerization, and recruitment of proteins including the MMPs coupled with their subsequent secretion, leading to ECM degradation at the invadopodial tip. As we determined above that MMP-2 was the main secreted MMP (Fig. 2), we initially examined the effect of sublethal doses of radiation (Fig. 6). Representa-
A
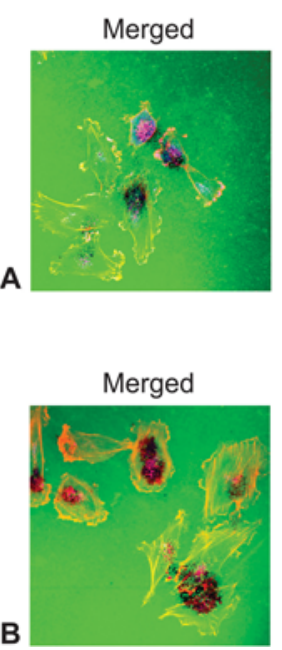

FITC

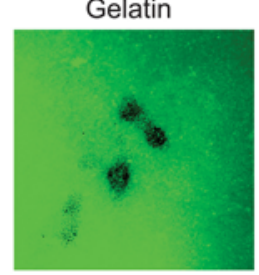

FITC

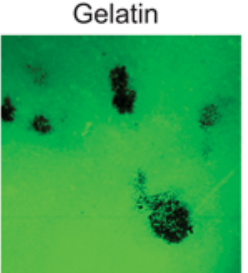

Rhodamine

Phalloidin

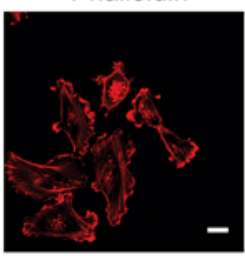

Rhodamine Phalloidin

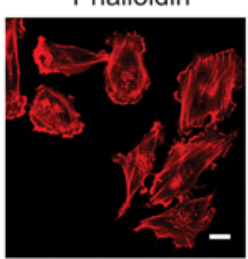

Rhodamine

Phalloidin

Cortactin

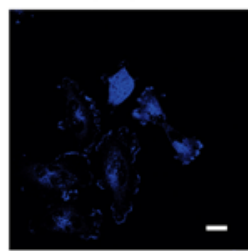

Tks5

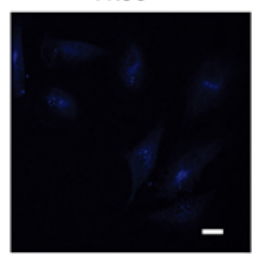

\& Cortactin

Rhodamine Phalloidin \& Tks5
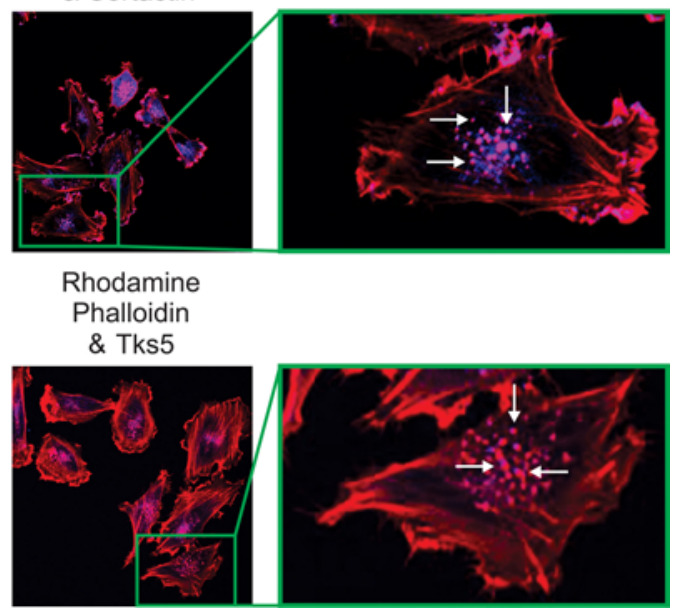

FIG. 4. Endogenous cortactin and Tks5 colocalize to invadopodia actin puncta in LN229 GBM cells. LN229 GBM cells were plated on coverslips coated with a thin film of cross-linked FITC-labeled gelatin. After 24 hours, the cells were fixed and stained for actin filaments with rhodamine-phalloidin (red), cortactin (A) or Tks5 (B) primary antibodies and an Alexa 405 secondary antibody. The white arrows indicate colocalization of rhodamine phalloidin-stained actin puncta with cortactin or Tks5 within invadopodia. The experiment was repeated twice, and representative images are shown. Scale bar $=20 \mu \mathrm{m}$. 
tive zymograms for analysis of culture medium taken from irradiated GBM cells 24 hours posttreatment are shown in Fig. 6A. We observed an increase in MMP-2 secretion and activation for U118 and U87MG GBM cells, in particular at $2.5 \mathrm{~Gy}$, with a slight increase in activated MMP-2 at 5 Gy. LN229 cells did not display a significant change. These results indicate that, overall, the doses of radiotherapy alone that were used in this study did not have a universal effect on MMP-2 secretion and activation for all cell lines. TMZ treatment $(0,200$, and $2000 \mu \mathrm{M})$ resulted in slight increases of secreted and activated MMP-2, especially at a concentration of $2000 \mu \mathrm{M}$ for the U118 glioma cell line (Fig. 7). As observed with radiotherapy alone, TMZ treatment alone did not result in a universal increase in MMP-2 secretion or activation. However, a combination of radiotherapy and TMZ treatment (Fig. 8) resulted in increased activation of MMP-2 for both U118 and LN229 glioma cells (2.5 Gy/1000 $\mu \mathrm{M}$ TMZ and $5 \mathrm{~Gy} / 1000 \mu \mathrm{M}$ TMZ). A slight decrease in activation of MMP-2 was observed at a combinatorial dose of $2.5 \mathrm{~Gy} / 1000 \mu \mathrm{M} \mathrm{TMZ}$; however, a recovery in secretion levels was then noted at a dose of 5 Gy/1000 $\mu \mathrm{M}$ TMZ.

\section{Invadopodia Activity Is Augmented by Sublethal Doses of Radiotherapy and TMZ}

As we had observed in the previous section that radiation or TMZ treatment could alter the MMP activity of the 3 GBM cell lines, we next examined whether it would ultimately also result in a functional change in invadopodia activity. The combined treatment of radiotherapy (5 Gy) and TMZ $(1000 \mu \mathrm{M})$ was observed to result in an increase in the invadopodia-mediated FITC-labeled gelatin degradation at 24 hours posttreatment in all 3 cell lines (Fig. 9A and B). While all 3 cell lines displayed this trend (5to 7-fold over the corresponding untreated controls), the U87MG and LN229 cell lines showed significant differences $(p<0.05)$ in their ability to degrade the FITC-labeled gelatin posttreatment. Importantly, while we observed an increase in the degree of degradation posttreatment of the GBM cells, we also detected a corresponding increase in the percentage of cells possessing FITC-labeled gelatindegrading invadopodia $(20 \%-25 \%$ increases over the corresponding untreated controls). Again, while this trend was present for all 3 cell lines, the U118 GBM cell line showed a significant difference $(\mathrm{p}<0.05)$ in the percentage of cells that formed invadopodia posttreatment. Even though the single-treatment doses employed in this study are higher than the daily doses used in the protocol of Stupp et al., 35 but lower than the total cumulative doses, these 2 results highlight important observations, suggesting that radiation and TMZ might induce both invadopodia activity (matrix degradation) and formation (percentage of cells forming invadopodia) in cells that survive treatment.

\section{Discussion}

Radiotherapy has remained one of the mainstays of GBM treatment for more than 30 years, ${ }^{5}$ and various therapeutics have been trialed in conjunction with radiotherapy. However, the landmark study by Stupp et al. observed that, since 2005, TMZ treatment was being used in conjunction
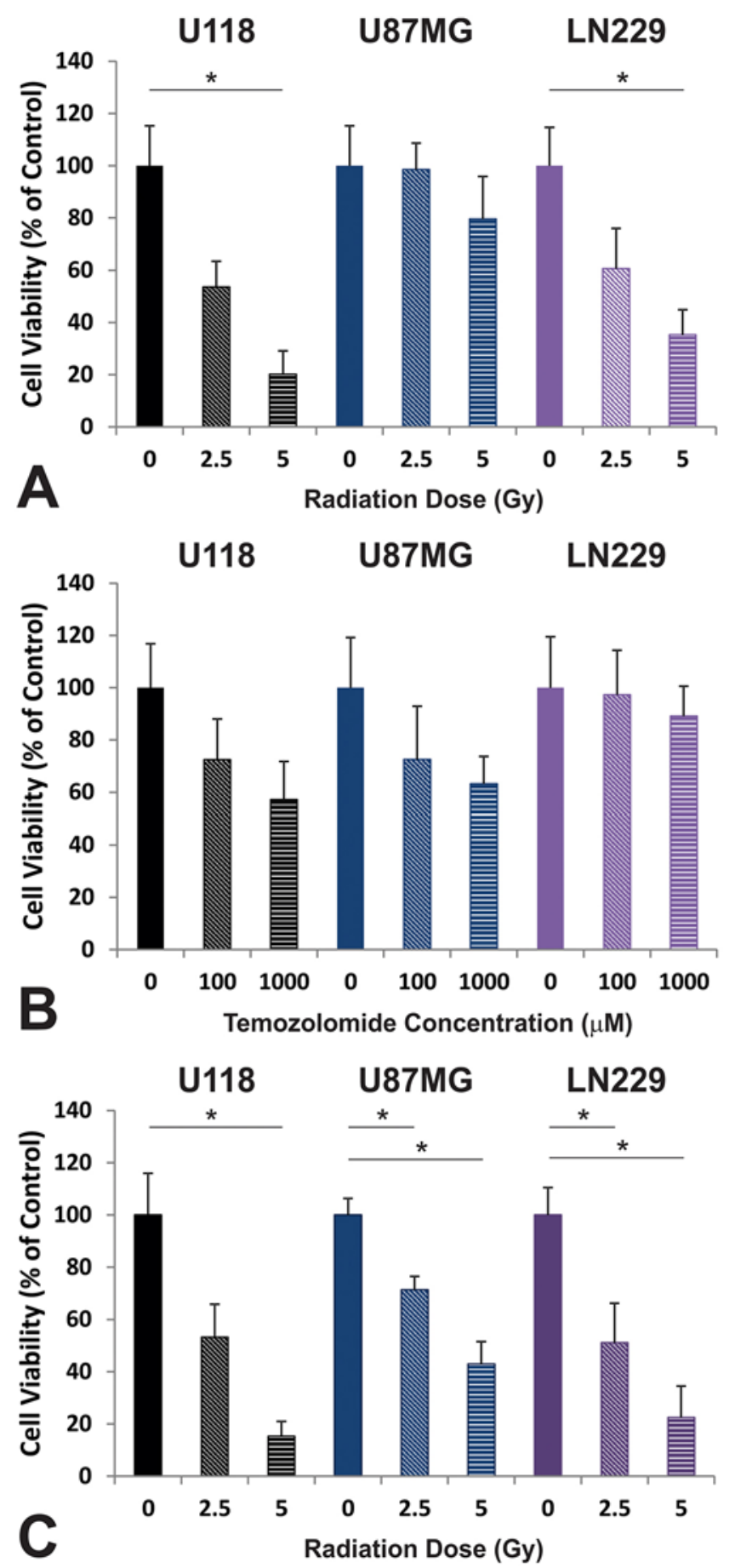

FIG. 5. GBM cell lines exhibit varying cell viability in response to radiotherapy or TMZ treatment. U118, U87MG, and LN229 GBM cell lines were plated in 96 -well plates and treated with varying doses of radiation $(0,2.5$, or $5 \mathrm{~Gy})(\mathrm{A})$, TMZ $(0,100$, or $1000 \mu \mathrm{M})(\mathrm{B})$, or a combination of radiation $(0,2.5$, or $5 \mathrm{~Gy})$ and $\mathrm{TMZ}(1000 \mu \mathrm{M})(\mathbf{C})$. Cell viability was determined at 72 hours after treatment. The experiment was repeated 3 times and the mean survival ( \pm standard error) relative to the control is shown in each panel. ${ }^{*} p<0.05$ versus control. Figure is available in color online only.

with surgery and radiotherapy. ${ }^{35}$ Despite an incremental increase in median survival to 15 months with this gold-standard protocol (2 Gy radiotherapy given 5 days per week 
$(\mathrm{kDa})$

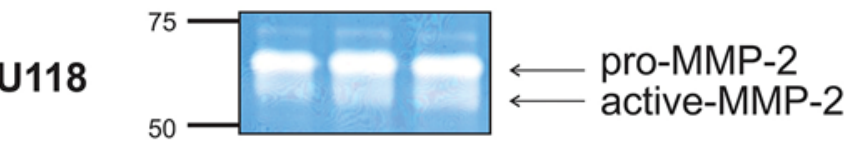

U87MG

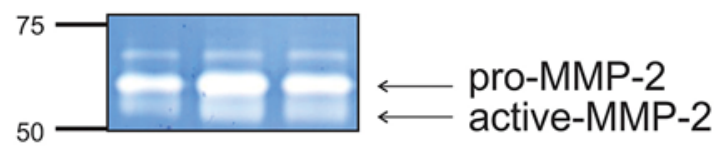

LN229

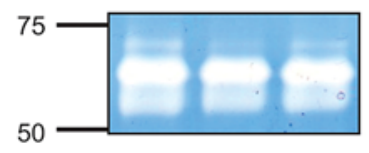
active-MMP-2
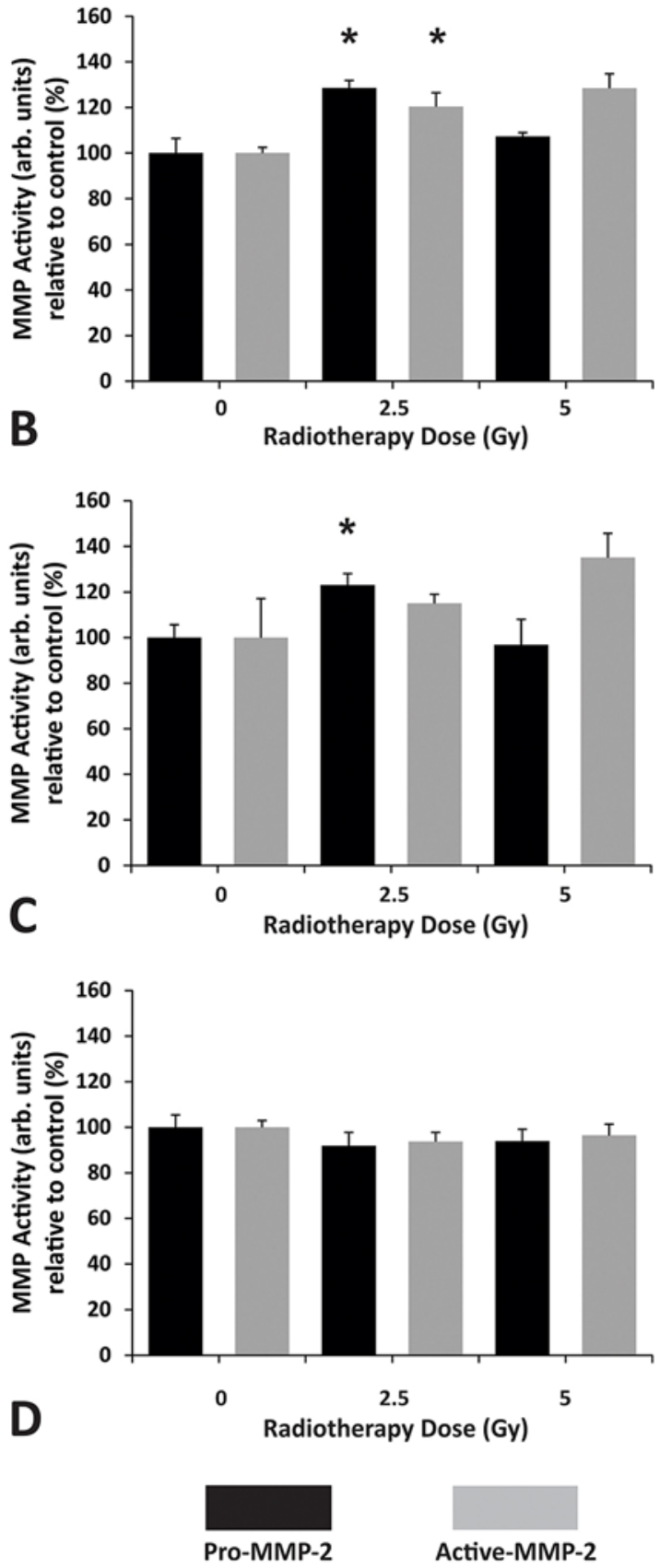

FIG. 6. Radiation treatment may alter MMP-2 secretion/activation in GBM cell lines. U118, U87MG, and LN229 GBM cell lines seeded in 6-well plates were treated with radiation at the following doses: 0, 2.5, or 5 Gy and subsequently placed in serum-free OptiMEM. At 24 hours, aliquots of the medium were taken and analyzed by gelatin-based zymography (A). The experiment was repeated 3 times, and the mean MMP activity relative to the control (analyzed via densitometry) is shown in each corresponding graph: U118 (B), U87MG (C), and LN229 (D). * $p<0.05$ versus control. arb. = arbitrary. Figure is available in color online only.

for 6 weeks - total of 60 Gy - and concomitant daily TMZ of $75 \mathrm{mg} / \mathrm{m}^{2}$ ) being used worldwide, there remains a continuing overall poor prognostic outcome for GBM patients, with only $26.5 \%$ of patients alive after 2 years. ${ }^{35}$ Therefore, there is a pressing need to understand the influence of this therapeutic approach on the cells that survive treatment.

It is known that tumor recurrence can be linked to the invasive tumor cell phenotype, allowing for the formation of tumor satellites at distant sites following clinical intervention, and ultimately thwarting the possibility of achiev- ing significant increases in patient survival. This is critical, as the combination of a radio- and chemotherapy approach is expected to primarily exert an antiproliferative effect by inhibiting DNA replication, with potentially only a minimal effect on the invasion process of any surviving cells. Importantly, it has been observed that the enhanced invasiveness of sublethally irradiated glioma cells is associated with increased MMP activity posttreatment. ${ }^{30,46}$ These observations, coupled with our previous research showing the relevance of invadopodia and their regulators in glio- 
$(\mathrm{kDa})$

U118

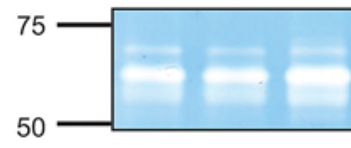

pro-MMP-2

$\longleftarrow$ active-MMP-2

U87MG

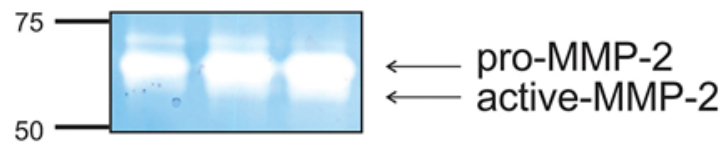

LN229

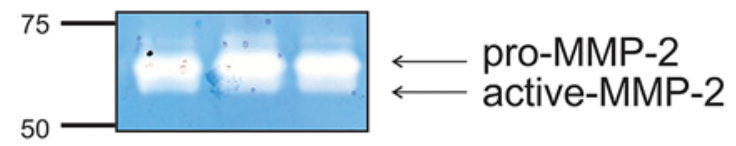

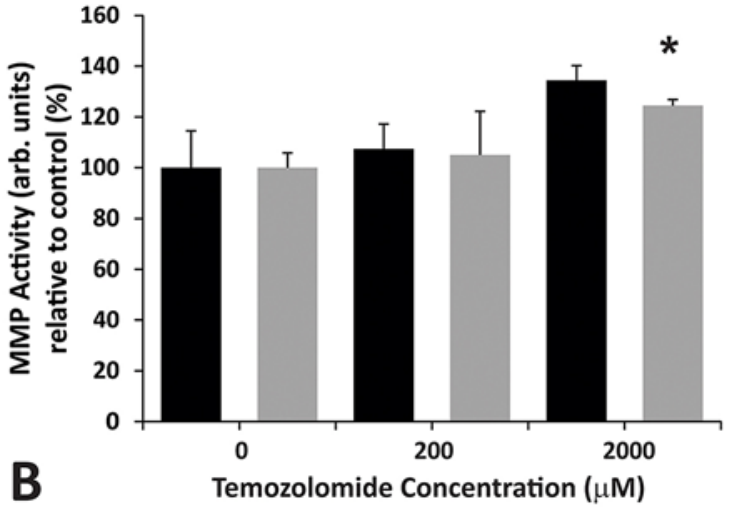
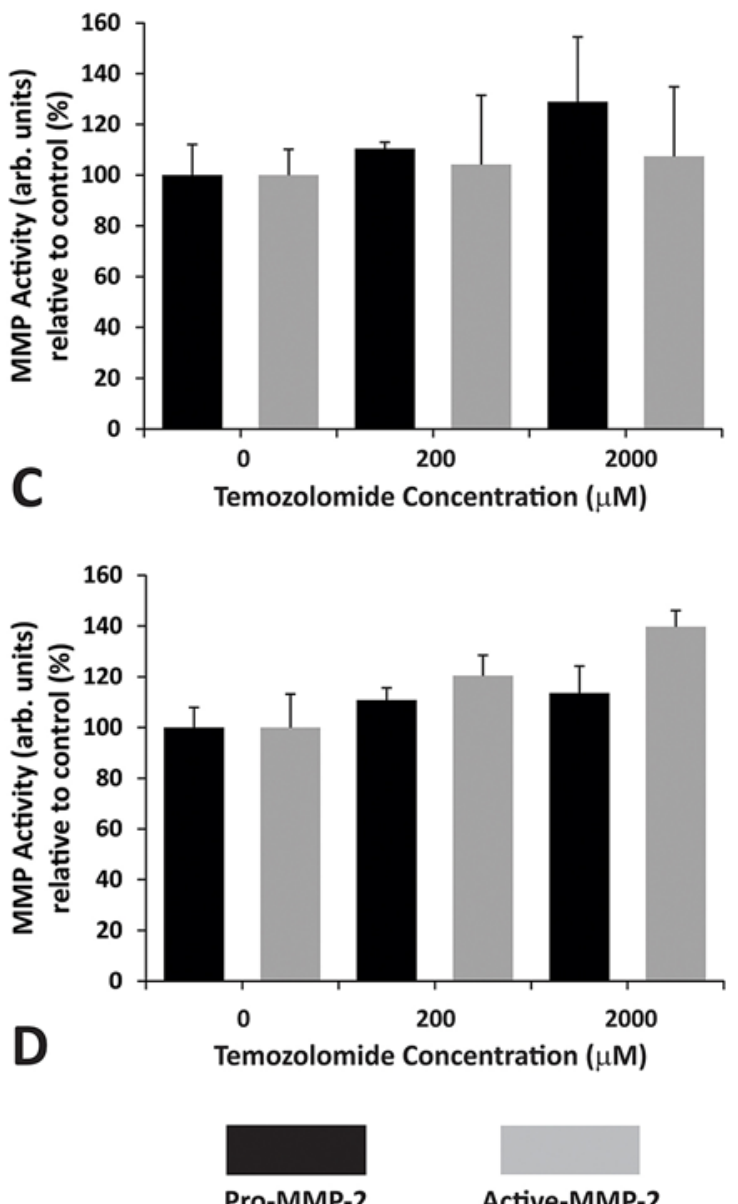

\section{Active-MMP-2}

FIG. 7. TMZ treatment does not have a significant impact on MMP-2 secretion/activation in GBM cell lines. U118, U87MG, and LN229 GBM cell lines seeded in 6-well plates were treated with TMZ for 24 hours at the following concentrations: 0, 200, or 2000 $\mu \mathrm{M}$. They were then incubated for an additional 24 hours in serum-free OptiMEM, after which aliquots of the medium were taken and analyzed by gelatin-based zymography (A). The experiment was repeated 3 times, and the mean MMP activity relative to the control (analyzed via densitometry) is shown in each corresponding graph: U118 (B), U87MG (C), and LN229 (D). ${ }^{*} p<0.05$ versus control. Figure is available in color online only.

ma, ${ }^{36,37}$ lead into our current study, in which we show that GBM cells that survive sublethal doses of radiation and TMZ, exhibit enhanced MMP-2 secretion and activation, combined with an increase in the formation and activity of invadopodia. While the complete mechanism leading to enhanced invadopodia activity remains unknown, the data presented suggest that there may be a rationale for explor- ing anti-invadopodia strategies for reducing the invasive potential of cells that are resistant to the current therapeutic protocols.

Cancer cells, including glioma, rely on a number of various invadopodia-related mechanisms to facilitate the dissemination of cells away from the primary tumor mass. These include cell adhesion to the ECM, degradation of 
$(\mathrm{kDa})$

U118

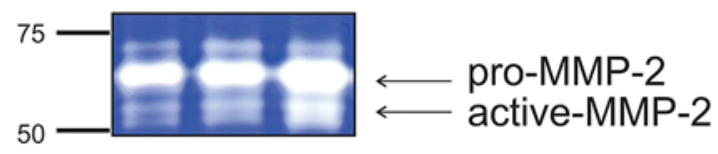

U87MG

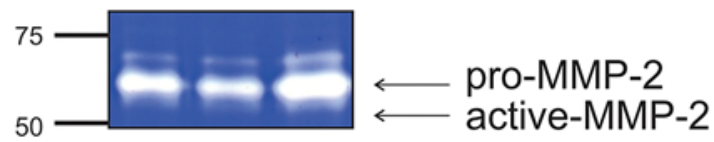

LN229

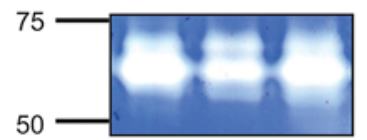

\section{$\longleftarrow$ pro-MMP-2} active-MMP-2
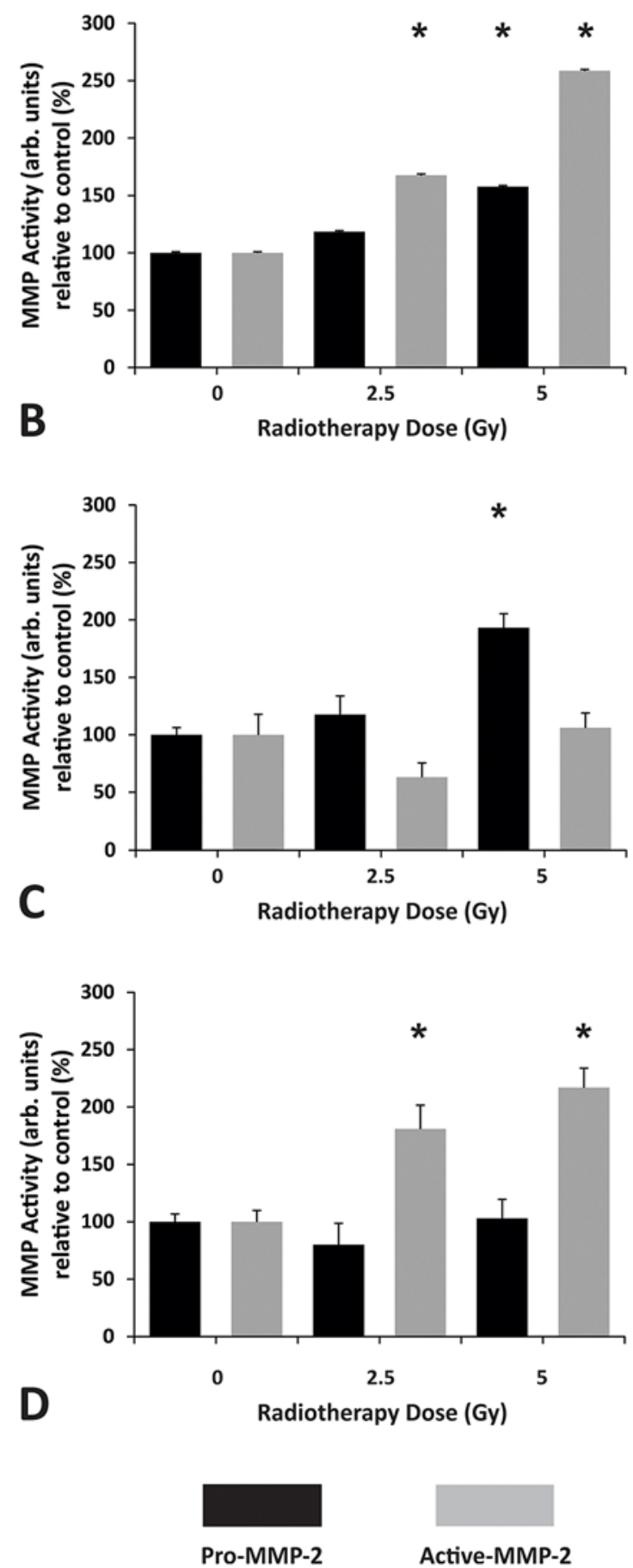

Active-MMP-2

FIG. 8. Radiation and TMZ treatment can enhance MMP-2 secretion/activation in GBM cell lines. U118, U87MG, and LN229 GBM cell lines seeded in 6-well plates were treated with radiation at the following doses: $0,2.5$, or 5 Gy and 24 hours postirradiation incubated with TMZ at a concentration of $1000 \mu \mathrm{M}$ in serum-free OptiMEM. At 24 hours, aliquots of the medium were taken and analyzed by gelatin-based zymography (A). The experiment was repeated 3 times, and the mean MMP activity relative to the control (analyzed via densitometry) is shown in each corresponding graph: U118 (B), U87MG (C), and LN229 (D). * $p<0.05$ versus control. Figure is available in color online only.

the ECM, and rearrangement of the cytoskeleton. ${ }^{24}$ Due to the emergence of invadopodia as facilitators of tumor cell invasion, studies are being carried out in laboratories in an attempt to uncover the relevant pathways involved in their biogenesis and how to combat the activity of invadopodia with compounds that target these relevant mecha- nisms. ${ }^{24}$ MMPs, as a class of zinc-dependent proteases, are known to degrade various membrane-related proteins, of which gelatin and collagen have been the most widely studied with respect to gliomas, ${ }^{4,31}$ especially as MMP-2 and MMP-9 are frequently overexpressed in the tumor tissue. ${ }^{4,31,42,44,48}$ Notably, the expression of MMPs, as regula- 

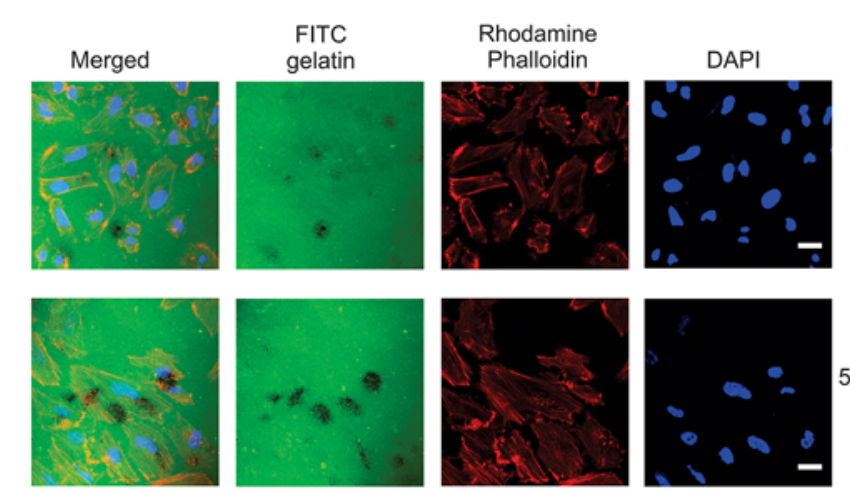

LN229

5 Gy radiotherapy $1000 \mu \mathrm{M}$ TMZ
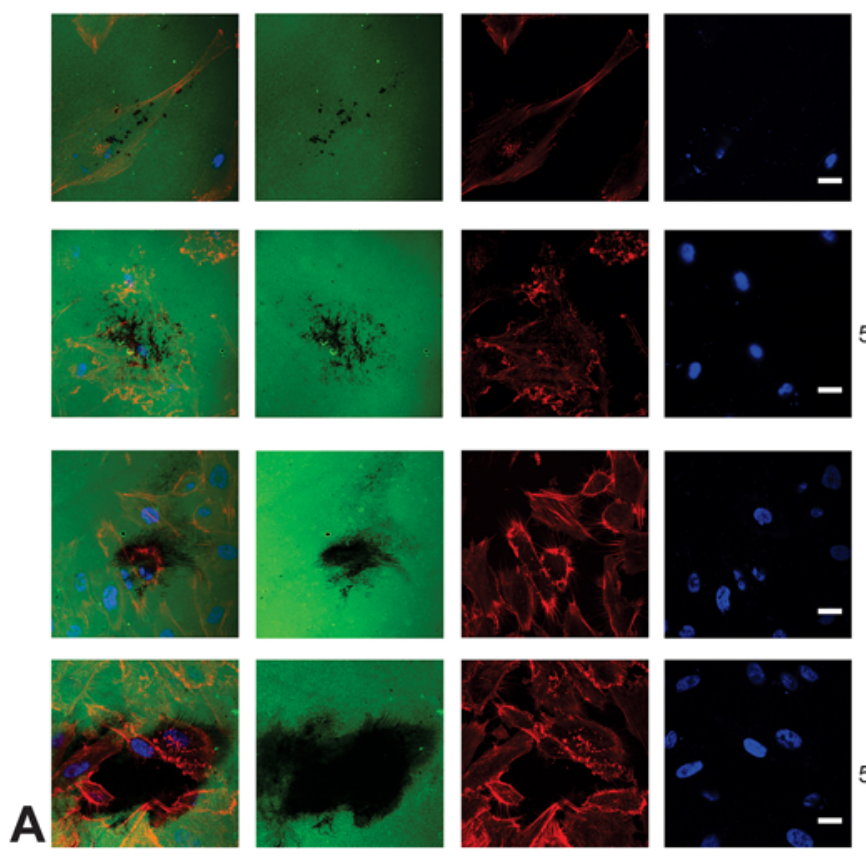

LN229

FIG. 9. Invadopodia activity in GBM cells is augmented at sublethal doses of radiation and TMZ. U118, U87MG, and LN229 GBM cell lines seeded in 6-well plates were treated with radiation at a dose of $5 \mathrm{~Gy}$ and after 8 hours were incubated with TMZ at a dose of $1000 \mu \mathrm{M}$. After an additional 24 hours, the cells were trypsinized and plated on coverslips coated with a thin film of cross-linked FITC-labeled gelatin. After 24 hours, the cells were fixed and stained for actin filaments with rhodamine-phalloidin (red) and DAPI as a nuclear stain. Degraded areas of FITC-labeled gelatin are evident as black areas devoid of FITC-labeled gelatin (green). DAPI staining of the nucleus is shown in blue. The experiment was repeated 3 times, and representative images (A), mean FITC-labeled gelatin degrading activity (B), and percentage of cells with FITC-labeled gelatin-degrading invadopodia (C) relative to the controls are shown. ${ }^{*} p<0.05$ versus control.

tors of invadopodia-mediated ECM degradation, can be upregulated upstream by numerous signaling molecules, such as Src and EGFR. There is evidence for the presence of an Src/EGFR-mediated phosphoinositide 3-kinase (PI3K)/ AKT pathway facilitating glioma cell invasion mediated via amplified MMP-2 secretion. ${ }^{29}$ This is significant, as the deregulation of EGFR-related pathways occurs frequently in many GBMs, which is also linked with EGFR amplification/overexpression and a poor patient prognosis. ${ }^{43} \mathrm{Im}$ portantly, studies have demonstrated that EGFR-triggered pathway activation can also confer resistance to radiation and chemotherapy, $3,8,11$ and this treatment resistance, leading to surviving cells, may also facilitate an increased invasive phenotype through the action of invadopodia in modifying and degrading the surrounding ECM.

Furthermore, EGF via binding to its receptor is known to be an inducer of invadopodia, ${ }^{16}$ and it is also recognized that downstream of this pathway, the common signaling hubs converge and involve Src and PI3Ks that can contribute to the formation and activity of invadopodia., ${ }^{7,37}$ Via its links to many signaling pathways, Src kinase phosphorylates a number of different invadopodia regulators, including Tks5. ${ }^{39}$ As there is evidence indicating that glioma cells can form invadopodia, ${ }^{10,37}$ our previous observations of the potential prognostic ability of Tks5 in glioma patient biopsies $^{36}$ and that the Src-mediated phosphorylation of Tks5 boosts the activation of MMP-2 ${ }^{38}$ imply that a link exists in the data obtained during our current study involving increased MMP-2 secretion/activation, FITC-labeled gelatin degradation, and the percentage of invadopodia-forming glioma cells in response to treatment. Of note, the information extracted from the independent Oncomine GBM data sets further highlights the relevance of invadopodia in GBM, where the overexpression of MMP-2 mRNA and 
other invadopodia regulators, such as Nck1, Src, Tks4, and Tks5, was detected in a number of the studies (Table 1). Intrinsic or acquired chemoresistance to TMZ is also an important factor hindering the successful treatment of glioma patients, with acquired resistance accounting for approximately $90 \%$ of recurrent diseases. ${ }^{27}$ Alterations to MGMT levels or mismatch repair status have been proposed as the main drivers for the development of this resistance, ${ }^{13}$ but activation of the Akt pathway has also been proposed and linked with Src levels..$^{45}$ A study by Sun et al. ${ }^{41}$ showed that TMZ-resistant glioma cells were associated with changes in actin cytoskeleton-related proteins and increased adhesion and migration properties that are known to be functionally linked to invadopodia-associated mechanisms. Therefore, the involvement of the EGFR-Src-(PI3)/AKT pathway in glioma invasion, invadopodia formation/activity, and treatment resistance (radiotherapy/TMZ) highlights the potential importance of this signaling axis as a therapeutic avenue in targeting glioma invasion through invadopodia after radiotherapy and TMZ treatment.

\section{Conclusions}

This current study has extended our previous findings, outlining the significance of invadopodia and their ability to mediate the invasive phenotype of GBM. We have now demonstrated that GBM cells that can survive sublethal single doses of radiotherapy and TMZ (which are higher than the daily protocol of Stupp et al ${ }^{35}$ of 2 Gy radiotherapy and concomitant daily $75 \mathrm{mg} / \mathrm{m}^{2} \mathrm{TMZ}$ ) display enhanced MMP-2 secretion and activation, which is critically also linked to an increase in the percentage of invadopodia forming glioma cells and FITC-labeled gelatin degradation. In summary, further research is required with respect to glioma invasion being therapeutically targeted through invadopodia-related mechanisms as an adjuvant to the current treatment approach of surgery, radiotherapy, and TMZ.

\section{Acknowledgments}

The study received funding from The Royal Melbourne Hospital Neuroscience Foundation

\section{References}

1. Artym VV, Zhang Y, Seillier-Moiseiwitsch F, Yamada KM, Mueller SC: Dynamic interactions of cortactin and membrane type 1 matrix metalloproteinase at invadopodia: defining the stages of invadopodia formation and function. Cancer Res 66:3034-3043, 2006

2. Beroukhim R, Getz G, Nghiemphu L, Barretina J, Hsueh T, Linhart D, et al: Assessing the significance of chromosomal aberrations in cancer: methodology and application to glioma. Proc Natl Acad Sci U S A 104:20007-20012, 2007

3. Bianco C, Tortora G, Bianco R, Caputo R, Veneziani BM, Caputo R, et al: Enhancement of antitumor activity of ionizing radiation by combined treatment with the selective epidermal growth factor receptor-tyrosine kinase inhibitor ZD1839 (Iressa). Clin Cancer Res 8:3250-3258, 2002

4. Binder DK, Berger MS: Proteases and the biology of glioma invasion. J Neurooncol 56:149-158, 2002

5. Bloom HJ: Combined modality therapy for intracranial tumors. Cancer 35:111-120, 1975
6. Bredel M, Bredel C, Juric D, Harsh GR, Vogel H, Recht LD, et al: High-resolution genome-wide mapping of genetic alterations in human glial brain tumors. Cancer Res 65:40884096, 2005

7. Buccione R, Caldieri G, Ayala I: Invadopodia: specialized tumor cell structures for the focal degradation of the extracellular matrix. Cancer Metastasis Rev 28:137-149, 2009

8. Chakravarti A, Chakladar A, Delaney MA, Latham DE, Loeffler JS: The epidermal growth factor receptor pathway mediates resistance to sequential administration of radiation and chemotherapy in primary human glioblastoma cells in a RAS-dependent manner. Cancer Res 62:4307-4315, 2002

9. Cheng L, Leibovich BC, Bergstralh EJ, Scherer BG, Pacelli A, Ramnani DM, et al: p53 alteration in regional lymph node metastases from prostate carcinoma: a marker for progression? Cancer 85:2455-2459, 1999

10. Chuang YY, Tran NL, Rusk N, Nakada M, Berens ME, Symons M: Role of synaptojanin 2 in glioma cell migration and invasion. Cancer Res 64:8271-8275, 2004

11. Ciardiello F, Caputo R, Borriello G, Del Bufalo D, Biroccio A, Zupi G, et al: ZD1839 (IRESSA), an EGFR-selective tyrosine kinase inhibitor, enhances taxane activity in bcl-2 overexpressing, multidrug-resistant MCF-7 ADR human breast cancer cells. Int J Cancer 98:463-469, 2002

12. Dong F, Eibach M, Bartsch JW, Dolga AM, Schlomann U, Conrad C, et al: The metalloprotease-disintegrin ADAM8 contributes to temozolomide chemoresistance and enhanced invasiveness of human glioblastoma cells. Neuro Oncol 17:1474-1485, 2015

13. Dullea A, Marignol L: MGMT testing allows for personalised therapy in the temozolomide era. Tumour Biol 37:8796, 2016

14. Giese A, Westphal M: Treatment of malignant glioma: a problem beyond the margins of resection. J Cancer Res Clin Oncol 127:217-225, 2001

15. Grelewski PG, Bar JK: The role of $\mathrm{p} 53$ protein and MMP2 tumor/stromal cells expression on progressive growth of ovarian neoplasms. Cancer Invest 31:472-479, 2013

16. Hayes KE, Walk EL, Ammer AG, Kelley LC, Martin KH, Weed SA: Ableson kinases negatively regulate invadopodia function and invasion in head and neck squamous cell carcinoma by inhibiting an HB-EGF autocrine loop. Oncogene 32:4766-4777, 2013

17. Jemal A, Siegel R, Xu J, Ward E: Cancer statistics, 2010. CA Cancer J Clin 60:277-300, 2010

18. Khosla D: Concurrent therapy to enhance radiotherapeutic outcomes in glioblastoma. Ann Transl Med 4:54, 2016

19. Kosztyla R, Chan EK, Hsu F, Wilson D, Ma R, Cheung A, et al: High-grade glioma radiation therapy target volumes and patterns of failure obtained from magnetic resonance imaging and ${ }^{18} \mathrm{~F}-\mathrm{FDOPA}$ positron emission tomography delineations from multiple observers. Int J Radiat Oncol Biol Phys 87:1100-1106, 2013

20. Lee J, Kotliarova S, Kotliarov Y, Li A, Su Q, Donin NM, et al: Tumor stem cells derived from glioblastomas cultured in bFGF and EGF more closely mirrored the phenotype and genotype of primary tumors than do serum-cultured cell lines. Cancer Cell 9:391-403, 2006

21. Li H, Wei M, Li S, Zhou Z, Xu D: Increased CD147 and MMP-9 expression in the normal rat brain after gamma irradiation. J Radiat Res (Tokyo) 54:27-35, 2013

22. Liang Y, Diehn M, Watson N, Bollen AW, Aldape KD, Nicholas MK, et al: Gene expression profiling reveals molecularly and clinically distinct subtypes of glioblastoma multiforme. Proc Natl Acad Sci U S A 102:5814-5819, 2005

23. Maire CL, Ligon KL: Molecular pathologic diagnosis of epidermal growth factor receptor. Neuro Oncol 16 (Suppl 8):viii1-viii6, 2014

24. Munson J, Bonner M, Fried L, Hofmekler J, Arbiser J, Bel- 
lamkonda R: Identifying new small molecule anti-invasive compounds for glioma treatment. Cell Cycle 12:2200-2209, 2013

25. Murat A, Migliavacca E, Gorlia T, Lambiv WL, Shay T, Hamou MF, et al: Stem cell-related "self-renewal" signature and high epidermal growth factor receptor expression associated with resistance to concomitant chemoradiotherapy in glioblastoma. J Clin Oncol 26:3015-3024, 2008

26. Murphy DA, Courtneidge SA: The 'ins' and 'outs' of podosomes and invadopodia: characteristics, formation and function. Nat Rev Mol Cell Biol 12:413-426, 2011

27. Oliva CR, Nozell SE, Diers A, McClugage SG III, Sarkaria JN, Markert JM, et al: Acquisition of temozolomide chemoresistance in gliomas leads to remodeling of mitochondrial electron transport chain. J Biol Chem 285:39759-39767, 2010

28. Ostrom QT, Gittleman H, Fulop J, Liu M, Blanda R, Kromer $\mathrm{C}$, et al: CBTRUS statistical report: primary brain and central nervous system tumors diagnosed in the United States in 2008-2012. Neuro Oncol 17 (Suppl 4):iv1-iv62, 2015

29. Park CM, Park MJ, Kwak HJ, Lee HC, Kim MS, Lee SH, et al: Ionizing radiation enhances matrix metalloproteinase-2 secretion and invasion of glioma cells through Src/epidermal growth factor receptor-mediated p38/Akt and phosphatidylinositol 3-kinase/Akt signaling pathways. Cancer Res 66:8511-8519, 2006

30. Pei J, Park IH, Ryu HH, Li SY, Li CH, Lim SH, et al: Sublethal dose of irradiation enhances invasion of malignant glioma cells through p53-MMP 2 pathway in U87MG mouse brain tumor model. Radiat Oncol 10:164, 2015

31. Raithatha SA, Muzik H, Muzik H, Rewcastle NB, Johnston RN, Edwards DR, et al: Localization of gelatinase-A and gelatinase-B mRNA and protein in human gliomas. Neuro Oncol 2:145-150, 2000

32. Rhodes DR, Yu J, Shanker K, Deshpande N, Varambally R, Ghosh D, et al: ONCOMINE: a cancer microarray database and integrated data-mining platform. Neoplasia 6:1-6, 2004

33. Shai R, Shi T, Kremen TJ, Horvath S, Liau LM, Cloughesy TF, et al: Gene expression profiling identifies molecular subtypes of gliomas. Oncogene 22:4918-4923, 2003 (Erratum in Oncogene 25:4256, 2006)

34. Stupp R, Brada M, van den Bent MJ, Tonn JC, Pentheroudakis G: High-grade glioma: ESMO Clinical Practice Guidelines for diagnosis, treatment and follow-up. Ann Oncol 25 (Suppl 3):iii93-iii101, 2014

35. Stupp R, Mason WP, van den Bent MJ, Weller M, Fisher B, Taphoorn MJ, et al: Radiotherapy plus concomitant and adjuvant temozolomide for glioblastoma. N Engl J Med 352:987-996, 2005

36. Stylli SS, I STT, Kaye AH, Lock P: Prognostic significance of Tks5 expression in gliomas. J Clin Neurosci 19:436-442, 2012

37. Stylli SS, Kaye AH, Lock P: Invadopodia: at the cutting edge of tumour invasion. J Clin Neurosci 15:725-737, 2008

38. Stylli SS, Luwor RB, Kaye AH, I STT, Hovens CM, Lock P: Expression of the adaptor protein Tks5 in human cancer: prognostic potential. Oncol Rep 32:989-1002, 2014

39. Stylli SS, Stacey TT, Verhagen AM, Xu SS, Pass I, Courtneidge SA, et al: Nck adaptor proteins link Tks5 to invadopodia actin regulation and ECM degradation. J Cell Sci 122:2727-2740, 2009
40. Sun L, Hui AM, Su Q, Vortmeyer A, Kotliarov Y, Pastorino $\mathrm{S}$, et al: Neuronal and glioma-derived stem cell factor induces angiogenesis with the brain. Cancer Cell 9:287-300, 2006

41. Sun S, Wong TS, Zhang XQ, Pu JK, Lee NP, Day PJ, et al: Protein alterations associated with temozolomide resistance in subclones of human glioblastoma cell lines. J Neurooncol 107:89-100, 2012

42. Tonn JC, Goldbrunner R: Mechanisms of glioma cell invasion. Acta Neurochir Suppl 88:163-167, 2003

43. Verhaak RG, Hoadley KA, Purdom E, Wang V, Qi Y, Wilkerson MD, et al: Integrated genomic analysis identifies clinically relevant subtypes of glioblastoma characterized by abnormalities in PDGFRA, IDH1, EGFR, and NF1. Cancer Cell 17:98-110, 2010

44. Wang L, Yuan J, Tu Y, Mao X, He S, Fu G, et al: Co-expression of MMP-14 and MMP-19 predicts poor survival in human glioma. Clin Transl Oncol 15:139-145, 2013

45. Wang Z, Sun J, Li X, Yang S, Yue S, Zhang J, et al: Downregulation of Src enhances the cytotoxic effect of temozolomide through AKT in glioma. Oncol Rep 29:1395-1398, 2013

46. Wild-Bode C, Weller M, Rimner A, Dichgans J, Wick W: Sublethal irradiation promotes migration and invasiveness of glioma cells: implications for radiotherapy of human glioblastoma. Cancer Res 61:2744-2750, 2001

47. Yamanaka R, Arao T, Yajima N, Tsuchiya N, Homma J, Tanaka R, et al: Identification of expressed genes characterizing long-term survival in malignant glioma patients. Oncogene 25:5994-6001, 2006

48. Zhao WJ, Zhang W, Li GL, Cui Y, Shi ZF, Yuan F: Differential expression of MMP-9 and AQP4 in human glioma samples. Folia Neuropathol 50:176-186, 2012

49. Zhou W, Xu Y, Gao G, Jiang Z, Li X: Irradiated normal brain promotes invasion of glioblastoma through vascular endothelial growth and stromal cell-derived factor $1 \alpha$. Neuroreport 24:730-734, 2013

\section{Disclosures}

The authors report no conflict of interest concerning the materials or methods used in this study or the findings specified in this paper.

\section{Author Contributions}

Conception and design: Stylli. Acquisition of data: Mao, Whitehead. Analysis and interpretation of data: Stylli, Mao, Whitehead. Drafting the article: Stylli, Mao, Whitehead, Morokoff, Luwor. Critically revising the article: Stylli, Mao, Whitehead, Kaye, Morokoff, Luwor. Reviewed submitted version of manuscript: Stylli, Mao, Morokoff, Luwor. Approved the final version of the manuscript on behalf of all authors: Stylli. Statistical analysis: Mao. Administrative/technical/material support: Paradiso, Kaye, Morokoff. Study supervision: Stylli.

\section{Correspondence}

Stanley S. Stylli, Department of Surgery, The University of Melbourne, The Royal Melbourne Hospital, Level 5, Clinical Sciences Building, Parkville, Victoria 3050, Australia. email: stanley. stylli@mh.org.au. 